\title{
ZEITSCHRIFT FÜR KRISTALLOGRAPHIE CRYSTALLINE MATERIALS
}
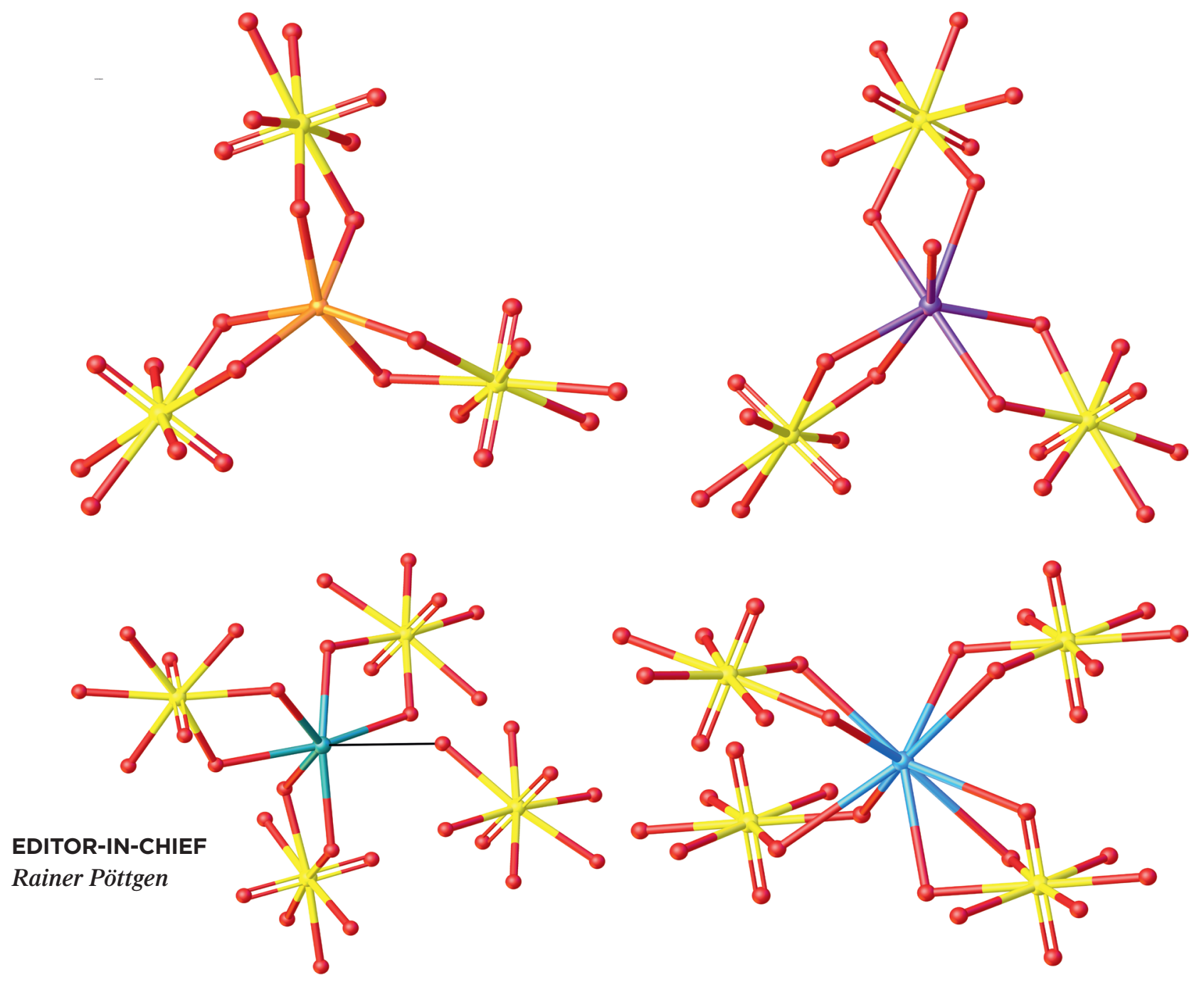


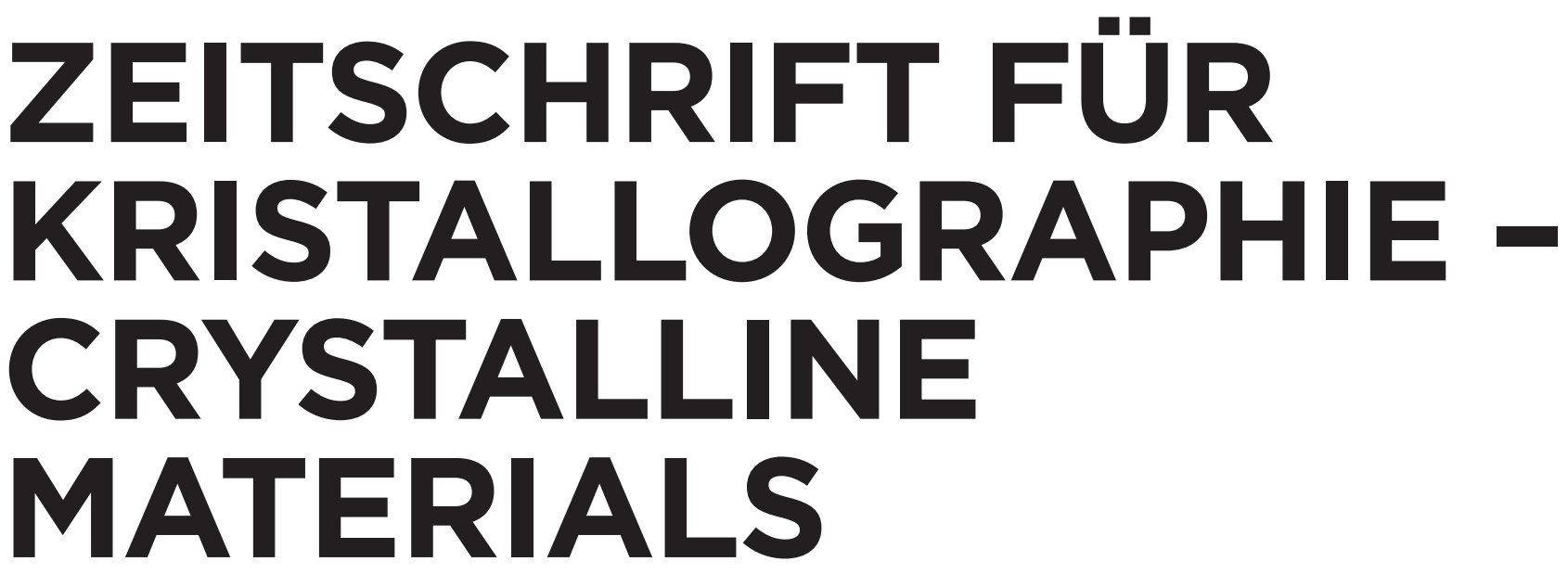

\section{EDITOR-IN-CHIEF}

Rainer Pöttgen, Münster, Germany

\section{EDITORS}

Evgeny Antipov, Moscow, Russia

Elena Boldyreva, Novosibirsk, Russian Federation

Karen Friese, Jülich, Germany

Hubert Huppertz, Innsbruck, Austria

Sandro Jahn, Köln, Germany

Edward R. T. Tiekink, Selangor Darul Ehsan, Malaysia

\section{ADVISORY BOARD}

José Antonio Alonso, Spain

Ross Angel, Italy

Daniel Chateigner, France

Xiangdong Ding, China

Herta Effenberger, Austria

Peter Gille, Germany

Sergey V. Krivovichev, Russia

Emil Makovicky, Denmark

Kunio Miki, Japan

Eric J. Mittemeijer, Germany

Rainer Niewa, Germany

Lars Öhrström, Sweden

Bogdan Palosz, Poland

Dhananjai Pandey, India

Thomas Proffen, USA

Jürgen Schreuer, Germany

Hiroyuki Takakura, Japan

Irena Wawrzycka-Gorczyca, Poland

Björn Winkler, Germany

Takamitsu Yamanaka, USA

\section{DE GRUYTER}


ABSTRACTED/INDEXED IN Baidu Scholar · Cabell's Directory · Chemical Abstracts Service (CAS): CAplus; SciFinder · Clarivate Analytics: Current Contents/Physical, Chemical and Earth Sciences; Journal Citation Reports/Science Edition; Science Citation Index; Science Citation Index Expanded; Web of Science · CNKI Scholar (China National Knowledge Infrastructure) · CNPIEC · Dimensions · EBSCO (relevant databases) - EBSCO Discovery Service · Elsevier: Chimica; Engineering Village; Reaxys; SCOPUS · Genamics JournalSeek · GeoRef · Google Scholar · Inspec · Japan Science and Technology Agency (JST) · J-Gate · JournalTOCs · KESLI-NDSL (Korean National Discovery for Science Leaders) · Microsoft Academic $\cdot$ Naviga (Softweco) · Paperbase · Pirabase · Polymer Library · Primo Central (ExLibris) · Publons · ReadCube · SCImago (SJR) · Summon (Serials Solutions/ ProQuest) $\cdot$ TDNet $\cdot$ TEMA Technik und Management $\cdot$ Ulrich's Periodicals Directory/ulrichsweb $\cdot$ WanFang Data $\cdot$ WorldCat $($ OCLC)

ISSN 2194-4946 · e-ISSN 2196-7105

All information regarding notes for contributors, subscriptions, back volumes and orders is available online at www.degruyter.com/zkri.

\section{EDITOR-IN-CHIEF}

Rainer Pöttgen, Universität Münster, Institut für Anorganische Chemie, Corrensstraße 30, 48149 Münster, Germany

Email: pottgen@uni-muenster.de; Fax: +49-2 51-83-3 6002

\section{EDITORS}

Evgeny Antipov, Moscow State University, Department of Chemistry, Moscow 119991, Russia

Email: antipov@icr.chem.msu.ru; Fax: +7-495-9 394788

Elena Boldyreva, Novosibirsk State University, ul. Pirogova, 2, Novosibirsk 630090, Russian Federation

Email: eboldyreva@yahoo.com

Karen Friese, Forschungszentrum Jülich, JCNS-2, PGI-4: Scattering Methods, 52425 Jülich, Germany

Email: k.friese@fz-juelich.de

Hubert Huppertz, University of Innsbruck, Institute of General, Inorganic and Theoretical Chemistry, Innrain 52a, 6020 Innsbruck, Austria

Email: hubert.huppertz@uibk.ac.at; Fax: +43-512-507-29 34

Sandro Jahn, Universität zu Köln, Mathematisch-Naturwissenschaftliche Fakultät, Institut für Geologie und Mineralogie, Zülpicher Str. 49 a, 50674

Köln, Germany

Email: s.jahn@uni-koeln.de; Fax: +49 221 470-4963

Edward R. T. Tiekink, Centre for Chemical Crystallography, Faculty of Science and Technology, Sunway University, 47500 Bandar Sunway, Selangor Darul Ehsan, Malaysia

Email: Edward.Tiekink@gmail.com

JOURNAL MANAGER Birgit Zoglmeier, De Gruyter, Rosenheimer Str. 143, 81671 München, Germany, Tel.: +49 (0)89 76902-426, Fax: +49 (0)89 76902-491, Email: birgit.zoglmeier@ degruyter.com

RESPONSIBLE FOR ADVERTISEMENTS Claudia Neumann, De Gruyter, Genthiner Straße 13, 10785 Berlin, Germany. Tel.: +49 (0)30 260 05-226, Fax: +49 (0) 30260 05-264, Email: anzeigen@ degruyter.com

(C) 2020 Walter de Gruyter GmbH, Berlin/Boston

TYPESETTING Compuscript Ltd., Shannon, Ireland

PRINTING Franz X. Stückle Druck und Verlag e.K., Ettenheim

COVER ILLUSTRATION Coordination geometries of alkali metal cations in the crystal structures of Na- (a), K- (b), Rb- (c) and Cs-bearing tri(acetato)uranylates (d). Legend: Na - orange; K - violet; Rb - cyan; Cs - blue, U - orange, O - red. (cf. Fig. 3 Kornyakov, Kalashnikova, Gurzhiy, Britvin, Belova and Krivovichev, pp. 95-103, this issue).

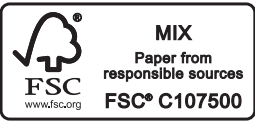

